\title{
Glucose-induced, cyclic-AMP-independent signalling pathway for activation of neutral trehalase in the fission yeast Schizosaccharomyces pombe
}

\author{
T. Soto, J. Fernandez, J. Cansado, J. Vicente-Soler and M. Gacto \\ Author for correspondence: M. Gacto. Tel: +3468 307100. Fax: +3468363963. \\ e-mail:maga@fcu.um.es
}

Department of Genetics and Microbiology, Facultad de Biología, University of Murcia, 30071 Murcia, Spain
The addition of glucose to derepressed cells of Schizosaccharomyces pombe provokes a CAMP signal and activation of the cytoplasmic neutral trehalase. This transduction pathway does not require functional RAS protein since RAS1disrupted cells exhibited a glucose response similar to that shown by control cells. Treatment of activated trehalase by alkaline phosphatase resulted in enzyme deactivation suggesting that trehalase may be modulated in vivo by reversible phosphorylation through CAMP-dependent protein kinase (PKA1). However, the addition of glucose to derepressed growing cells of Schiz. pombe lacking the catalytic subunit of protein kinase A (Apka1::URA4 ${ }^{+}$strains) induced stimulation of trehalase as well as phosphorylation of the enzyme protein. This glucose-induced response was absent in PKA 1-deficient cells from resting cultures. Addition of exogenous CAMP activated trehalase in normal growing cells but failed to produce any effect on trehalase in PKA1-disrupted growing cells. These results confirm the occurrence of a PKA1-dependent pathway for trehalase activation and imply the existence of another glucoseinduced phosphorylation pathway capable of activating trehalase during growth by a distinct, CAMP-independent protein kinase. At least one of the upstream components playing a role in the transduction of this alternative signal is either absent or inactive in cells from stationary phase and sporulated cultures. Cells harbouring the disrupted PKA1 gene responded also to a heatshock signal by increasing trehalase activity, thus revealing that this enzyme may be a target common to various signalling pathways in the fission yeast.

Keywords: trehalase, glucose signal, cyclic AMP, $p k a 1$ mutants, fission yeast

\section{INTRODUCTION}

Evidence has recently accumulated to show that in Schizosaccharomyces pombe cAMP serves as second messenger in signalling pathways that control key cellular processes in response to nutritional conditions. The initiation of mating and meiosis (De Voti et al., 1991; Mochizuki \& Yamamoto, 1992) and the expression of the glucose-repressible gene FBP1 (Hoffman \& Winston, 1991), which encodes fructose-1,6-bisphosphatase, are examples of physiologically relevant mechanisms controlled by cAMP. In these cases, the activation of cAMPdependent protein kinase (protein kinase A; PKA1) produced by an increase in the level of the cyclic nucleotide likely promotes phosphorylation of intermediate transducing elements that are inhibitory for both the initiation of sexual development and the transcription of the FBP1 gene.

Another cAMP-dependent pathway, somehow similar to one present in the budding yeast Saccharomyces cerevisiae (Thevelein, 1991), has also been implicated in the posttranslational regulation of neutral trehalase in the fission yeast (Carrillo et al., 1992, 1994). This enzyme is involved in the mobilization of the storage disaccharide trehalose which, in turn, determines viability of yeast cells under a variety of stress conditions (Van Laere, 1989; De Virgilio et al., 1990). The input cAMP-mediated signal that triggers trehalase activation is induced by addition of glucose or other compounds to derepressed cells, and reversible protein phosphorylation is viewed as the mechanism responsible for transduction of such a signal 
into the activation response (Carrillo et al., 1994). Repressed cells lack this glucose-induced signal (Carrillo et al., 1992).

In Schiz. pombe the catalytic subunit of PKA1 is encoded by a unique gene (Maeda et al., 1994; Yu et al., 1994). To understand better the process of glucose-induced trehalase activation in this organism we have analysed the activation response in cells with a defective cAMP-dependent pathway, unable to transduce the glucose-induced cAMP signal beyond the PKA1 level due to the absence of the $P K A 1$ gene product. The results demonstrate the existence of a new glucose-induced phosphorylation pathway in derepressed growing cells that may lead from glucose addition to trehalase activation without concomitant PKA1 activity. Moreover, the results support that trehalase is a natural substrate for PKA1 and may be a target common to at least another protein kinase present in the fission yeast.

\section{METHODS}

Yeast strains and media. Yeast strains used in this study are listed in Table 1. They are derivatives of those originally described by Leupold (1950). The strains were cultured at $28^{\circ} \mathrm{C}$ with shaking in liquid medium containing either $2 \%(\mathrm{w} / \mathrm{v})$ glucose or $2 \%(\mathrm{w} / \mathrm{v})$ glycerol as a carbon source supplemented with $0.6 \%$ yeast extract. In the first case, the cells were collected by centrifugation from derepressed stationary-phase cultures after glucose exhaustion. When cultured on glycerol, derepressed cultures were harvested in the mid-exponential phase of growth. Sporulation in strain L968 was induced on MEL liquid medium containing $3 \%(\mathrm{w} / \mathrm{v})$ malt extract. In all cases, after growth the cells were washed and resuspended in $10 \mathrm{mM} \mathrm{MES} / \mathrm{KOH}$ buffer, $\mathrm{pH} 6.0$, at a concentration of $50 \mathrm{mg}$ wet wt $\mathrm{ml}^{-1}$ (approximately $8 \times 10^{8}$ cells $\mathrm{ml}^{-1}$ ).

Trehalase activation and enzyme assays. Suspensions of derepressed cells from growing or resting cultures were incubated in a shaking water bath and allowed to equilibrate at $30{ }^{\circ} \mathrm{C}$ for $5 \mathrm{~min}$. After addition of $100 \mathrm{mM}$ glucose or $5 \mathrm{mM}$ cAMP (zero time), samples were removed at timed intervals and the cells washed as above. Cell extracts were obtained as previously indicated and the supernatant fluids used for trehalase assays and protein determination (Carrillo et al., 1992). For activation of trehalase upon heat shock, the procedure of De Virgilio et al. (1990) was followed except that both repressed and derepressed growing cultures were employed with similar results. 1,2-Dioctanoyl-sn-glycerol, oleic acid, neomycin and staurosporin were used as described by Brandao et al. (1994).

Table 1. Schiz. pombe strains used

\begin{tabular}{|c|c|c|}
\hline Strain & Genotype & Reference \\
\hline L972 & $b^{-}$prototroph & Leupold (1950) \\
\hline L975 & $b^{+}$prototroph & Leupold (1950) \\
\hline L968 & $b^{90}$ prototroph & Leupold (1950) \\
\hline RP91 & $b^{+} \Delta \operatorname{ras} 1:: L E U 2^{+}$leu1 & Fukui et al. (1989) \\
\hline Eg545 & $b^{+} \Delta m a t 2,3:: L E U 2^{+}$leu $1^{+}$ & Nielsen et al. (1992) \\
\hline JZ633 & $\begin{array}{l}b^{90} \text { ade6-M216 leu1 ura4-D18 } \\
\Delta p k a 1:: U R A 4^{+}\end{array}$ & Maeda et al. (1994) \\
\hline JZ636 & $\begin{array}{l}b^{+} \text {ade6-M210 leu1 ura4-D18 } \\
\Delta p k a 1:: U R A 4^{+}\end{array}$ & Maeda et al. (1994) \\
\hline
\end{tabular}

Trehalase activity is given as enzyme units ( $\mathrm{mg}$ protein $)^{-1}$. One unit is $1 \mathrm{nmol}$ glucose released $\min ^{-1}$ under the stated assay conditions.

Reproducibility of results. All experiments were repeated at least three times with comparable results. Representative results are shown.

\section{RESULTS}

\section{Glucose-induced activation of trehalase in RAS- disrupted cells of Schiz. pombe}

We have described previously that the addition of glucose to wild-type derepressed cells of Schiz. pombe during exponential or stationary phase resulted in a cAMP peak and subsequent activation of trehalase (Carrillo et al., 1994). This response appears to be somehow similar to that ensuing glucose addition to derepressed cells of Sac. cerevisiae (Thevelein, 1991). However, in contrast to the glucose-induced pathway for trehalase activation present in the budding yeast, which requires at least one of the two functional RAS proteins present in those cells, the corresponding single homologous RAS1 protein is not needed in the fission yeast for transduction of the glucose signal. Following glucose addition, derepressed cells from either exponential- or stationary-phase cultures of the RAS1-disrupted strain RP91 of Schiz. pombe exhibited trehalase activation which was comparable to that shown by the closely related $\mathrm{ras}^{+}$strain Eg545 (Fig. 1) or the wild-type strains L972, L975 and L968 (not shown). This is consistent with the assumption that RAS does not act as modulator of adenylate cyclase in the fission yeast (Fukui et al., 1986).

\section{CAMP-induced activation of trehalase in PKA1- disrupted cells of Schiz. pombe}

In growing cells of Schiz. pombe exogenous cAMP produces a sustained activation of trehalase, and this stimulation saturates at about $5 \mathrm{mM}$ cAMP (Carrillo et al., 1992). Addition of this nucleotide to control strain L975

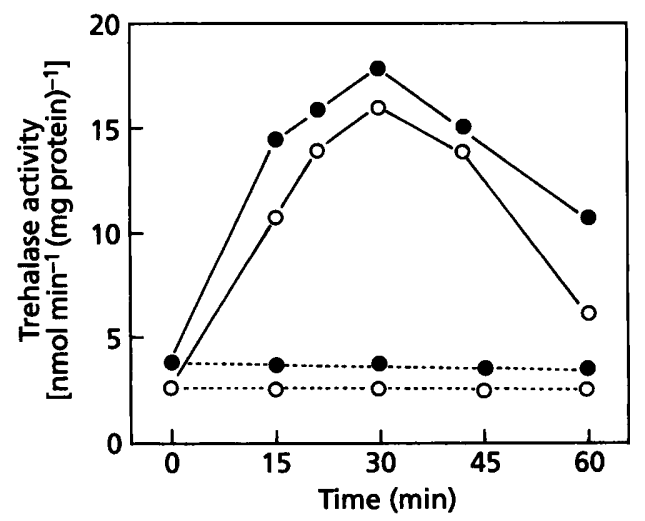

Fig. 1. Activation of trehalase in derepressed growing cells of a ras $^{+}$strain (Eg545, O) and a ras strain (RP91, O) of Schiz. pombe after addition of $100 \mathrm{mM}$ glucose at time zero. The cultures were grown on glycerol and harvested in exponential phase. Trehalase activity in control cells without addition is represented by a discontinuous line. 


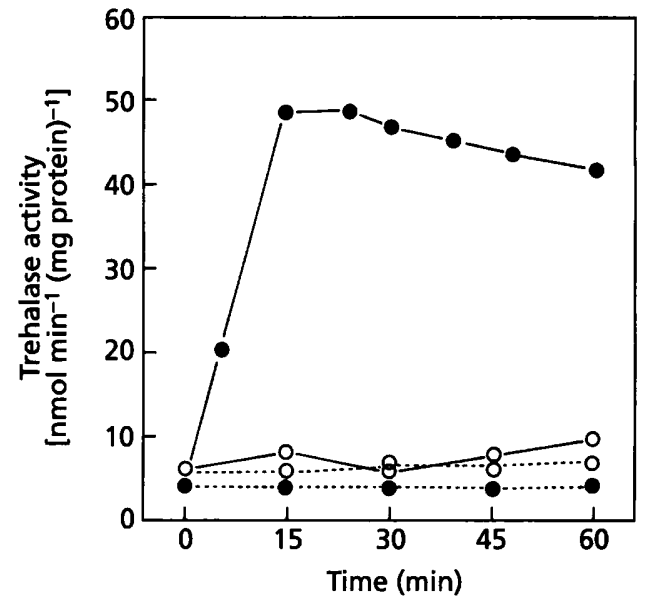

Fig. 2. Effect of the addition of CAMP on trehalase from derepressed cells of Schiz. pombe growing in medium containing glycerol. Trehalase activity was measured in cells of wild-type strain L975 (pka 1+, O) or strain JZ636 (pka1, O) after addition of $5 \mathrm{mM}$ CAMP and theophylline at time zero. Trehalase activity in control cells without addition is represented by a discontinuous line.

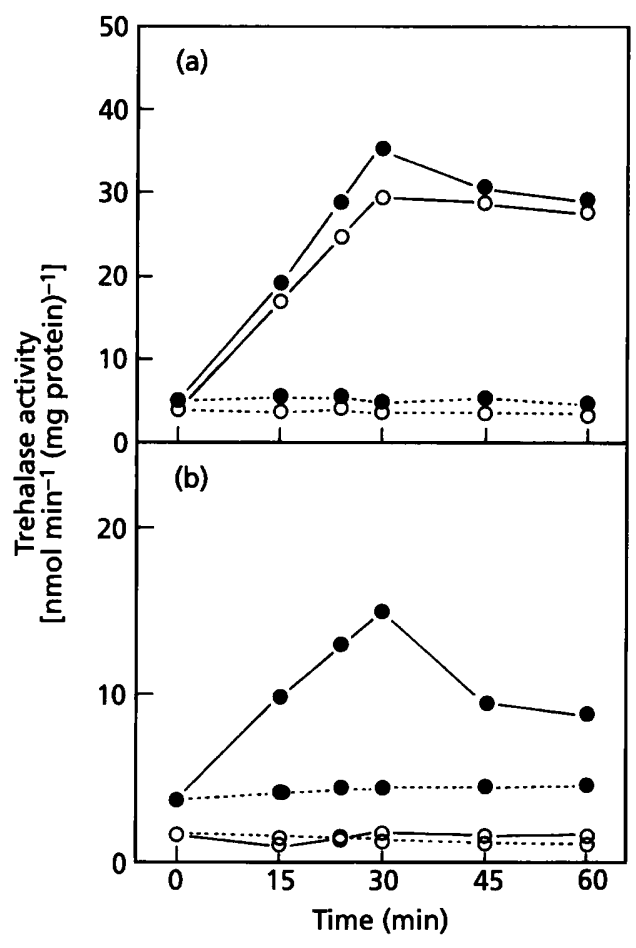

Fig. 3. Glucose-induced activation of trehalase in derepressed growing (a) or stationary-phase cultures (b) of strain $L 975$ (pka1+, O) and strain JZ636 (pka1, O). Cells were grown in either glycerol- or glucose-containing medium until exhaustion of the sugar. Trehalase activity in the control cells is represented by a discontinuous line.

cells resulted in trehalase activation whereas cAMP was without effect when added to cells of the haploid strain JZ636 which contain a disruption in the $P K A 1$ gene encoding the catalytic subunit of protein kinase A (Fig. 2)
Table 2. Deactivation of trehalase by phosphatase

Alkaline phosphatase from calf intestine $[100$ units ( $\mathrm{mg}$ cell extract $\left.)^{-1}\right]$, free of proteinase activity, was incubated in $0.1 \mathrm{M}$ Tris/ $\mathrm{HCl}, \mathrm{pH} 8$, with enzyme preparations from either derepressed growing cells pulsed with $100 \mathrm{mM}$ glucose for $15 \mathrm{~min}$ or repressed growing cells after treatment at $40^{\circ} \mathrm{C}$ for $60 \mathrm{~min}$. After $10 \mathrm{~min}$ at $37^{\circ} \mathrm{C}$ the remaining trehalase activity was determined.

\begin{tabular}{|lllcc|}
\hline Strain & $\begin{array}{c}\text { Activating } \\
\text { agent }\end{array}$ & $\begin{array}{c}\text { Trehalase activity } \\
\text { [units (mg protein) }\end{array}$ & $\begin{array}{c}\text { Deactivation } \\
\text { (\%) }\end{array}$ \\
\cline { 3 - 3 } & & Control & \\
\hline \multirow{2}{*}{ L975 } & Glucose & $10 \cdot 8$ & $3 \cdot 2$ & $71 \cdot 4$ \\
& Heat shock & $50 \cdot 6$ & $23 \cdot 9$ & $52 \cdot 8$ \\
JZ636 & Glucose & $14 \cdot 8$ & $2 \cdot 5$ & $83 \cdot 1$ \\
& Heat shock & $75 \cdot 5$ & 34.0 & $55 \cdot 0$ \\
\hline
\end{tabular}

(Maeda et al., 1994). These results confirm the existence of a cAMP-dependent pathway for trehalase activation and the idea that $P K A 1$ is the only gene encoding the catalytic subunit of protein kinase A in Schiz. pombe.

\section{Glucose-induced activation of trehalase in PKA1- disrupted cells of Schiz. pombe}

Earlier reports on the analysis of trehalase activation by glucose in the fission yeast proposed a single cAMPmediated pathway regulating trehalase activity (Carrillo $e t$ al., 1994). It was surprising, therefore, to find that the blockage of this signalling pathway in derepressed cells of strain JZ636 did not prevent the glucose-induced response provided these cells were growing (Fig. 3a). Interestingly, such glucose-induced activation of trehalase was not seen in $P K A 1$-disrupted resting cells (Fig. 3b). These unexpected observations revealed an alternative, previously unnoticed, pathway for trehalase activation independent of the cAMP-PKA1-mediated cascade.

The activation of trehalase that follows glucose addition to control and $P K A 1$-deficient growing cells corresponds to phosphorylation of the enzyme protein since the increased activity was in both cases sensitive to phosphatase treatment in vitro (Table 2). Thus, the data are indicative of the presence in growing cells of another glucose-controlled protein kinase(s) able to suppress $P K A 1$ deficiency in the signalling process for trehalase activation. Similar results to those shown for the JZ636 strain were obtained by using another Scbiz. pombe PKA1disrupted strain. The self-conjugating strain JZ633 is derepressed for sexual development even in the presence of rich nutrition (Maeda et al., 1994) so that upon culture in liquid medium sporulation readily occurs. Asci and spores, which in part germinate to conjugate again, coexist with vegetative cells in cultures of this strain until exhaustion of the carbon source. Although disruption of $P K A 1$ alone causes a slower vegetative growth, glucose was able to induce activation of trehalase in growing 


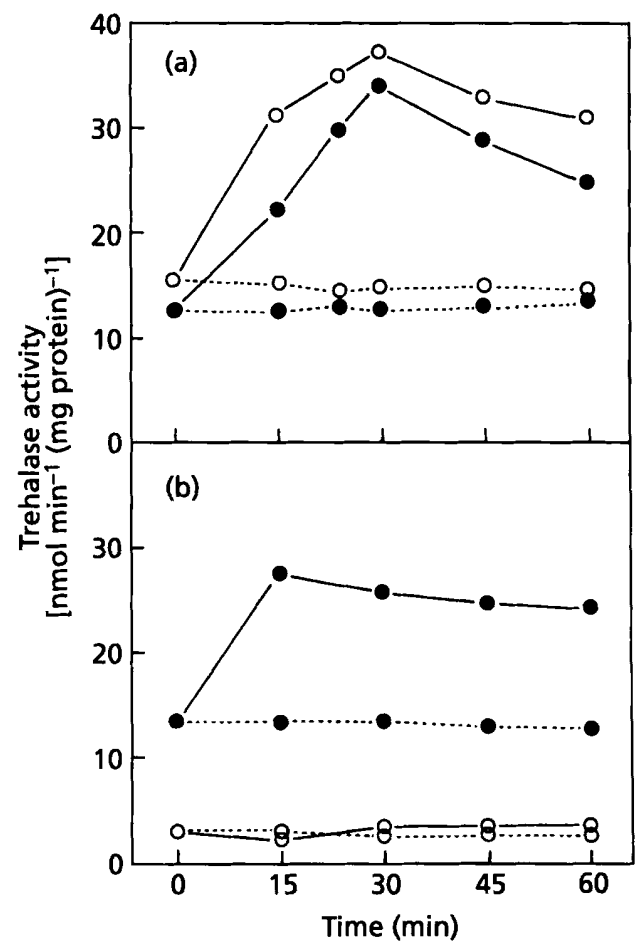

Fig. 4. Glucose-induced activation of trehalase in derepressed growing (a) or stationary-phase cultures (b) of strain L968 (pka1+, o) and strain JZ633 (pka1, O). Sporulated resting cultures were obtained in MEL medium. Trehalase activity in the control cells is represented by a discontinuous line.

cultures of this strain (Fig. 4a). In contrast, the effect of glucose was again null in strain JZ633 cells from stationary-phase cultures (Fig. 4b).

Since cells from stationary-phase cultures of strains JZ636 and JZ633 lacked glucose-induced activation of trehalase it seems reasonable to assume that neither of the two pathways referred to above is operative in resting pka1 cells. Therefore, some physiological impairment affecting the alternative (PKA1-independent) pathway for the activation response must be present in these cells in addition to the genetic disruption of the PKA1 gene. In contrast, control cells from resting cultures showed glucose-induced enzyme activation (Figs $3 \mathrm{~b}$ and $4 \mathrm{~b}$ ), indicating that the PKA1-mediated pathway is the only operative for trehalase activation in stationary-phase cells.

On the other hand, as trehalase was activated by glucose in both growing and resting $R A S$-disrupted cells (see above), the involvement of the RAS protein in any of the two glucose-induced signalling pathways for trehalase activation was discarded.

\section{Effect of some protein kinase C modulators on trehalase}

Addition of well-known activators of mammalian protein kinase $C$, such as $2 \mathrm{mM}$ 1,2-dioctanoyl-sn-glycerol or oleic acid (Berridge, 1987), to either normal or PKA1-

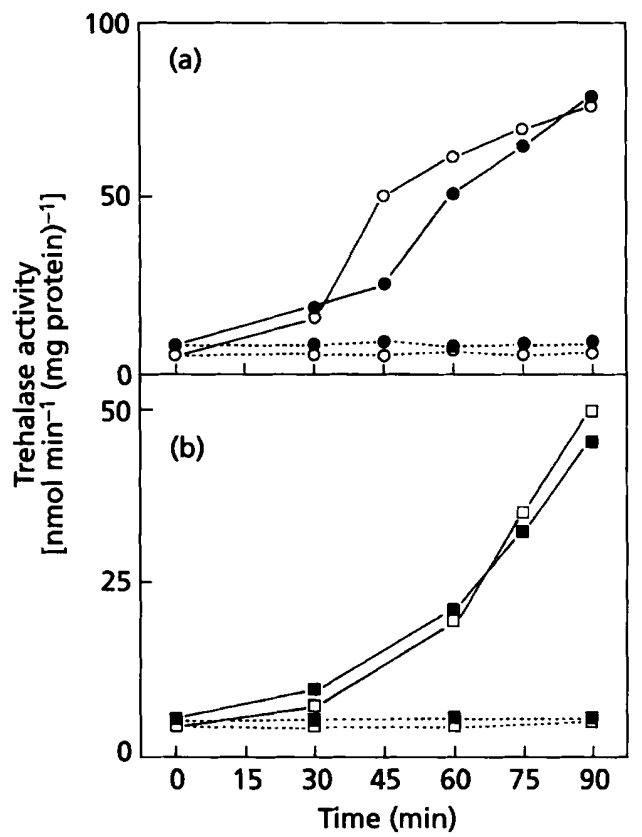

Fig. 5. Trehalase activity in repressed (a) or derepressed cells (b) of Schiz. pombe during a heat shock. Cultures growing on glucose or glycerol were either shifted at zero time to $40^{\circ} \mathrm{C}$ or maintained at $28^{\circ} \mathrm{C}$ as controls. Strains: L975 (pka 1+, O), JZ636 (pka1, 0), Eg545 (ras, 0 ), RP91 (ras, $\square)$. Trehalase activity in control cultures is represented by a discontinuous line.

disrupted cells of the fission yeast did not affect basal levels of trehalase. Also, preincubation with $1 \mu \mathrm{M}$ staurosporine, a potent inhibitor of protein kinase $C$ in mammalian cells (Tamakori et al., 1986), or $0.1 \mathrm{mM}$ neomycin, which acts as a strong inhibitor of the phosphatidylinositol pathway (Shimma \& Uno, 1990), did not prevent a subsequent glucose-induced activation of trehalase (not shown). These results imply that the protein kinase which activates trehalase in PKA1disrupted cells following the addition of glucose differs from both PKA1 and C-type protein kinases.

\section{Additive effect of glucose and thermal shock on activation of trehalase}

Trehalase can be enhanced by heat shock in growing cells of strain L972, as well as in other wild-type strains, in the presence of cycloheximide (De Virgilio et al., 1990). We have determined that this activation mechanism does not use cAMP as a second messenger (Carrillo et al., 1994). Congruent with this conclusion, $P K A 1$-disrupted strains of Schiz. pombe exhibited a heat-shock-induced activation of trehalase similar to that shown by their control isogenic counterparts (Fig. 5a). Also, like the glucose-induced signalling pathways, this heat-shock-induced pathway appeared to be independent of RAS function (Fig. 5b) and to involve phosphorylation of trehalase (Table 2). Moreover, unlike those induced by glucose, this activation pathway functions in both repressed (De Virgilio et al., 1990) and derepressed growing cells (Fig. 6). Taken 


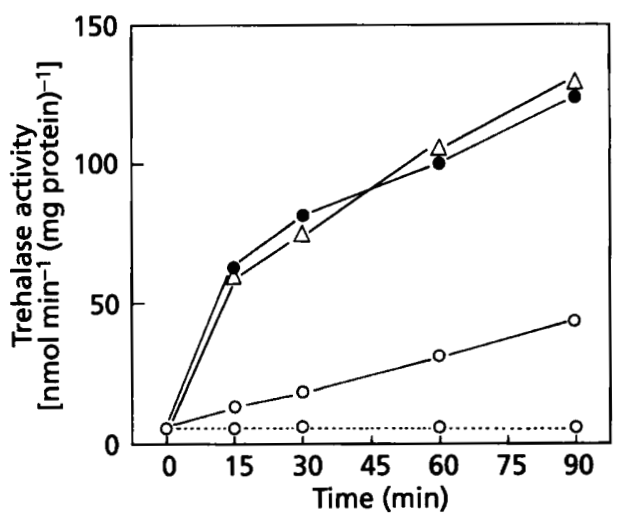

Fig. 6. Activation of trehalase by heat shock and addition of glucose. Strain L972 was grown at $30^{\circ} \mathrm{C}$ on glycerol-containing medium till mid-exponential phase and then shifted to $40^{\circ} \mathrm{C}$ at time zero with $(O)$ or without $(O)$ the simultaneous addition of $100 \mathrm{mM}$ glucose. At the times shown cell samples were withdrawn from the shifted culture not supplemented with glucose and then pulsed with $100 \mathrm{mM}$ glucose for $30 \mathrm{~min}$ as indicated in Methods except that the activation induced by the sugar was performed at $40^{\circ} \mathrm{C}(\Delta)$. Trehalase in control cultures maintained at $30^{\circ} \mathrm{C}$ is represented by a discontinuous line.

together, these observations imply that activation of trehalase in heat-shocked cells of Schiz. pombe involves a phosphorylation pathway different from those induced by glucose.

By using a well-characterized wild-type strain we tried to show that trehalase activation in Scbiz. pombe may in fact serve as a reporter for the function of more than just one signal transduction pathway. We assumed that under saturated conditions $\left(100 \mathrm{mM}\right.$ glucose or $\left.40^{\circ} \mathrm{C}\right)$ the glucose- or heat-shock-induced pathways were fully operative and that the resulting trehalase activity would then reflect the ability for activation corresponding to those particular pathways. By this reasoning, a further stimulation by glucose or heat shock of already activated trehalase would imply the existence of another independent activation pathway. As shown in Fig. 6, trehalase activated in heat-shocked cells from growing cultures was additionally activated after supplement with glucose and both effects were rather additive, i.e. the activation specifically due to heat shock and that due to glucose was about the same upon simultaneous or independent induction. These results indicate that glucose can tune trehalase in parallel to the heat-shock-induced activation of the enzyme and might serve to indicate the existence of different pathways of activation with trehalase as a common target substrate.

\section{DISCUSSION}

Previous attempts to obtain deactivation of trehalase from Schiz. pombe by phosphatase treatment were unsuccessful and thus the mechanism of post-translational regulation of neutral trehalase remained to be elucidated (De Virgilio et al., 1991). We now report that under appropriate conditions (Table 2) changes in trehalase activity are produced by phosphatase and thus that phosphorylation

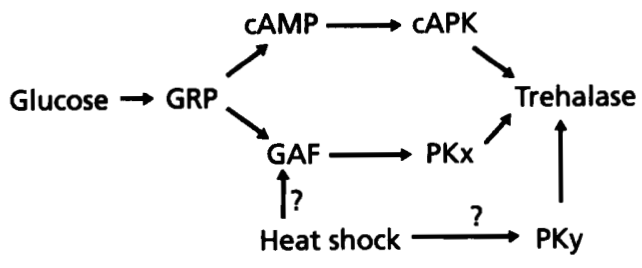

Fig. 7. Partial scheme of the proposed flow of events leading to glucose-induced activation of trehalase in Schiz. pombe. The tentative model does not exclude the involvement of additional elements, not represented, in the transmission of the input signals. GRP, glucose-repressible protein; GAF, growthassociated factor; CAPK, CAMP-dependent protein kinase or PKA1; PKx,y, undetermined protein kinase(s).

of trehalase likely occurs during activation of the enzyme whether induced by glucose or heat shock.

Also, this study shows that a scheme for glucose-induced trehalase activation which has at least two confluent pathways is more appropriate than that suggested previously by Carrillo et al. (1994). Summarizing previous and present data, the control of trehalase activation in Schiz. pombe is outlined in Fig. 7. The $P K A 1$ gene product controls trehalase activity, but at least one other protein kinase appears to perform a similar role. The current interpretation of our observations is that the cooperation of two independent glucose-induced signalling pathways, one including the intermediate activation of adenylate cyclase and another hitherto unknown, requiring alternative transduction elements which may be inactive or absent in growth-arrested cells, ensures trehalase activation in derepressed growing cells after addition of glucose. Although pka1 strains provide substantial evidence to support the idea of a glucose-induced cAMPindependent activation of trehalase, we have no insight so far as to the biochemical nature of the reaction giving rise to the transient character of this alternative response.

Glucose was able to bypass the blockage in the transmission of the signal caused by the disruption of the $P K A 1$ gene only in derepressed growing cells of the $p k a 1$ strains and not in the same cells at stationary phase. These results support the idea that the glucose induction of the alternative (PKA1 independent) phosphorylation pathway does not merely require derepression conditions but also cell growth. This observation stresses differences between derepressed growing and resting cells and points to a fundamental growth-related requirement for the induction of the cAMP-independent activation response of trehalase. It appears unlikely that the factor required is just ATP needed for enzyme phosphorylation, instead of some upstream growth-associated transducing element(s), since wild-type resting cells are able to phosphorylate trehalase through the cAMP-dependent pathway upon arrival of glucose.

Two major questions remain to be determined. One is whether the two glucose-induced signalling pathways are simultaneously functional in derepressed growing cells. Although not fully established, our results are in agreement with the idea that the glucose signal is preferentially 
transduced throughout the PKA1-independent pathway during growth since no substantial difference in enzyme activity was found between normal and $P K A 1$-disrupted cells after trehalase activation by the sugar (Figs $3 a$ and 4a). The second question is whether the heat-shockinduced pathway shares transduction elements of the glucose-induced PKA1-independent pathway for trehalase activation. So far, arguments for or against this possibility can be drawn. In fact, growth is required in both cases for induction of the signalling pathways but the results summarized in Fig. 6 rather favour two independent activation pathways without common intermediates. Further studies should be informative about these possibilities.

Schiz. pombe contains a diversity of kinases associated with different processes (Herskowitz, 1995). In this context, it is intriguing that there are close similarities between the conditions required for the activation of trehalase through the alternative (PKA1-independent) glucose-induced or the heat-shock-induced phosphorylation and those that are operative during control of other kinase-related processes in the fission yeast. For instance, the product of gene $\mathrm{R} A N 1$ (also called PAT1), which plays an important role in switching between vegetative growth and growth arrest, is a protein kinase distinct from protein kinase A, but both share some properties and common substrates. The RAN1 kinase is normally essential for vegetative growth (Beach et al., 1985; McLeod \& Beach, 1986), and its absence provokes entry into stationary phase, sexual conjugation and commitment of the cell to meiosis. Overstimulation of PKA1 by cAMP inhibits normal meiosis and allows vegetative cell division to occur in the absence of the $\mathrm{R} A N 1$ gene product (Beach $e t$ al., 1985). Also, overexpression of $P K A 1$ has an ability to suppress the loss of function of the $\mathrm{R} A N 1$ gene (Maeda et al., 1994), implying that there is some overlap in the function of PKA1 and the product of the RAN1 gene. This genetic interaction of PKA1 with RAN1 implies that some substrates common to both kinases can be phosphorylated during vegetative growth and not during meiotic initiation. Our results do not attempt to establish a link between RAN1 and trehalase activation. However, because the requirement for growth in the glucose- or heat-shock-induced trehalase activation of pka1 strains somehow mirrors the function during vegetative growth of RAN1 protein kinase activity and its inactivation in resting cells, it is tempting to speculate that a RAN1-like protein kinase might be involved in the alternative cAMPindependent pathways for trehalase stimulation. Although their various components remain to be determined, the results presented support the view that trehalase in the fission yeast is a target common to various signalling pathways involved in environmental monitoring.

\section{ACKNOWLEDGEMENTS}

We are grateful to Drs M. Yamamoto (Tokyo), O. Nielsen (Copenhagen), A. Duran and S. Moreno (Salamanca) for kind provision of Scbiz. pombe strains used in this study. We also thank F. Garro for technical help.

\section{REFERENCES}

Beach, D., Rodgers, L. \& Gould, J. (1985). R $A N 1^{+}$controls the transition from mitotic division to meiosis in fission yeast. Curr Genet 10, 297-311.

Berridge, M. J. (1987). Inositol trisphosphate and diacylglycerol: two interacting second messengers. Annu Rev Biochem 56, 159-163.

Brandao, R. L., Magalhaes-Rocha, N. M., Alijo, B., Ramos, J. \& Thevelein, J.M. (1994). Possible involvement of a phosphatidylinositol-type signaling pathway in glucose-induced activation of plasma membrane $\mathrm{H}^{+}$-ATPase and cellular proton extrusion in the yeast Saccharomyces cerevisiae. Biochim Biopbys Acta 1223, 117-124.

Carrillo, D., Vicente-Soler, J. \& Gacto, M. (1992). Activation of neutral trehalase by fermentable sugars and cAMP in the fission yeast Schizosaccharomyces pombe. FEMS Microbiol Lett 98, 61-66.

Carrillo, D., Vicente-Soler, J. \& Gacto, M. (1994). Cyclic AMP signalling pathway and trehalase activation in the fission yeast Schizosaccharomyces pombe. Microbiology 140, 1467-1472.

De Virgilio, C., Simmen, U., Hottiger, T., Boller, T. \& Wiemken, A. (1990). Heat shock induces enzymes of trehalose metabolism, trehalose accumulation and thermotolerance in Schizosaccharomyces pombe, even in the presence of cycloheximide. FEBS Lett 273, 107-110.

De Virgilio, C., Muller, J., Boller, T. \& Wiemken, A. (1991). A constitutive, heat shock-activated neutral trehalase occurs in Schizosaccharomyces pombe in addition to the sporulation-specific acid trehalase. FEMS Microbiol Lett 84, 84-90.

De Voti, J., Seydoux, G., Beach, D. \& McLeod, M. (1991). Interaction between $\mathrm{ran}^{+}$protein kinase and cAMP dependent protein kinase as negative regulators of fission yeast meiosis. EMBO J 10, 3759-3768.

Fukui, Y., Kozasa, T., Kajiro, Y., Takeda, T. \& Yamamoto, M. (1986). Role of a ras homologue in the life cycle of Scbizosaccharomyces pombe. Cell 44, 329-336.

Fukui, Y., Miyake, S., Satoh, M. \& Yamamoto, M. (1989). Characterization of the Schizosaccharomyces pombe ral2 gene implicated in activation of the ras1 gene product. Mol Cell Biol 9, 5617-5622.

Herskowitz, I. (1995). MAP kinase pathways in yeast: for mating and more. Cell 80, 187-197.

Hoffman, C. S. \& Winston, F. (1991). Glucose repression of transcription of the Schizosaccharomyces pombe $f b p 1$ gene occurs by a cAMP signaling pathway. Genes \& Dev 5, 561-571.

Leupold, U. (1950). Die Vererbung von Homothallie und Heterothallie bei Schizosaccharomyces pombe. C R Trav Lab Carlsberg Ser Pbysiol 24, 381-480.

Maeda, T., Watanabe, Y., Kunitomo, H. \& Yamamoto, M. (1994). Cloning of the pka1 gene encoding the catalytic subunit of the cAMP-dependent protein kinase in Scbizosaccharomyces pombe. J Biol Chem 269, 9632-9637.

McLeod, M. \& Beach, D. (1986). Homology between the $\operatorname{ran} 1^{+}$gene of fission yeast and protein kinases. EMBO J 5, 3665-3671.

Mochizuki, N. \& Yamamoto, M. (1992). Reduction of the intracellular cAMP level triggers initiation of sexual development in fission yeast. Mol \& Gen Genet 233, 17-24.

Nielsen, O., Davey, J. \& Egel, R. (1992). The ras1 function of Schizosaccharomyces pombe mediates pheromone-induced transcription. EMBO J 11, 1391-1395.

Shimma, Y. \& Uno, I. (1990). Isolation and characterization of neomycin-sensitive mutants in Saccharomyces cerevisiae. $J$ Gen Microbiol 136, 1753-1761. 
Tamakori, T., Nomoto, H., Takahashi, I., Kato, Y., Monimoto, M. \& Tomita, F. (1986). Staurosporine, a potent inhibitor of phospholipid/ $\mathrm{Ca}^{++}$dependent protein kinase. Biochem Biophys Res Commun 135, 397-402.

Thevelein, J. M. (1991). Fermentable sugars and intracellular acidification as specific activators of the RAS-adenylate cyclase signalling pathway in yeast: the relationship to nutrient-induced cell cycle control. Mol Microbiol 5, 1301-1307.
Van Laere, A. (1989). Trehalose, reserve and/or stress metabolite? FEMS Microbiol Rev 63, 201-210.

Yu, G., Li, J. \& Young, D. (1994). The Schizosaccharomyces pombe ptea1 gene, encoding a homolog of cAMP-dependent protein kinase. Gene 151, 215-220.

Received 27 March 1995; revised 6 June 1995; accepted 27 June 1995. 